\title{
SERUM INOCULATION AS A POSSIBILITY FOR ELIMINATION OF PORCINE REPRODUCTIVE AND RESPIRATORY SYNDROME (PRRS) FROM A FARROW-TO-FINISH PIG FARM
}

\author{
Marina ŠTUKELJ ${ }^{1 *}$, Jan PLUT ${ }^{1}$ and Ivan TOPLAK ${ }^{2}$ \\ ${ }^{1}$ Institute for the Health Care of Pigs and ${ }^{2}$ Institute for Microbiology and Parasitology, \\ Veterinary Faculty, University of Ljubljana, Gerbičeva 60, 1000 Ljubljana, Slovenia
}

(Received 2 April 2015; accepted 22 June 2015)

\begin{abstract}
The large heterogeneity among porcine reproductive and respiratory syndrome virus (PRRSV) isolates is probably the main obstacle to its effective control using current commercial vaccines. Intentionally exposing all breeding pigs to PRRSV circulating on the farm could eliminate porcine reproductive and respiratory syndrome (PRRS) from the herd. The objective of this study was to eliminate PRRS from a farrow-to-finish pig farm by serum inoculation. The owner was acquainted with the strict biosecurity measures. Breeding pigs were immunised with serum, which was obtained from PRRSV-positive weaners from the same farm. The percent of antibody high positive breeding pigs decreased six months after serum inoculation, while 34 months after serum inoculation no more antibody high positive pigs were detected and $56.8 \%$ of breeding pigs and all other categories were free of antibodies. In the breeding herd no virus was detected during all testing while PRRSV circulated in 2-month-old weaners until 12 months after serum inoculation. Later all tested samples from weaners, growers and fatteners were negative. Herd closure and the adoption of strict biosecurity measures are essential. Serum inoculation of the breeding herd proved to be a successful measure for eliminating PRRS from this farrow-to-finish farm.
\end{abstract}

Key words: Porcine, PRRS, serum inoculation, immunisation, elimination

Porcine reproductive and respiratory syndrome (PRRS) is a viral infection endemic in most swine-producing countries (Dee et al., 2001). The costs of the disease have been calculated in the USA, the Netherlands, Denmark and Sweden. Costs per sow in acute outbreaks range from $100 €$ in Europe to over $200 €$ in the USA (Holtkamp et al., 2013). Recent calculations from Denmark show that the yearly financial losses for the Danish swine industry caused by endemic or chronic PRRSV infections are around 15 million $€$ in the most probable scenario, ranging from 4 to 43 million $€$ depending on different assumptions (Duinhof et

*Corresponding author; E-mail: marina.stukelj@vf.uni-lj.si; Phone: 00386 (1) 477-9206; Fax: 00386 (1) 477-9880 
al., 2014). The total cost for the US swine industry was estimated to be 530 million $€$ each year (Holtkamp et al., 2013). The disease is characterised by reproductive failure, including late-term abortions, early farrowing, stillbirths, weakborn piglets, and increased mortality in neonates, nursing and growing pigs (Zimmerman et al., 2012). PRRSV infection is difficult to control due to the large heterogeneity among the isolates. A variety of strategies have been described for PRRS elimination, including total depopulation/repopulation and partial depopulation (Dee et al., 1993), isowean (Gramer et al., 1999) or segregated early weaning (Rajic et al., 2001), test and removal (Fano et al., 2005) and mass vaccination with unidirectional pig flow and herd closure (Torremorell and Christianson, 2002). Elimination of disease results in the disappearance of all clinical cases of a specified disease (Toma et al., 1991), which is the consequence of no virus replication and circulation in the population of pigs. No single strategy for elimination will work on infected farms; therefore, the individual programme must be implemented on the basis of the unit's pig flow and facility design as well as the serological results (Gillespie and Caroll, 2003).

PRRS elimination is the long-term goal, but the first step is stabilisation of the breeding herd. Herd stability is defined as a herd with uniform titres of antibodies against PRRSV and without virus (Gillespie and Caroll, 2003). Stabilisation can be achieved by the immunisation of the breeding herd using commercial vaccines, serum inoculation or natural exposure (Fano et al., 2005). It appears that currently available vaccines may not be effective in protecting against infections with genetically different strains of PRRSV (Meng, 2000; Kimman et al., 2009). Numerous studies have shown some cross-protection against different strains which is reflected only in the reduction of clinical signs and lesions without eliminating the virus (Murtaugh and Genzow, 2011). Inoculation with a homologous strain provides a high level of protection against the same or a highly similar virus (Shibata et al., 2000; Batista et al., 2002). It is, however, accepted that homologous immunity is more protective than heterologous immunity. Serum inoculation is the intentional immunisation of pigs with the strain of PRRS virus originating from the same, infected farm (homologous herd strain). The method consists of intramuscular injection of all pigs in the breeding herd with serum derived from acutely infected pigs (Fano et al., 2005). Shibata et al. (2000) showed that after exposure to a homologous PRRSV strain, pigs did not develop clinical signs, and virus replication was reduced in titre and length of infection.

Herd closure is an important action in an infected herd to achieve herd stability. In the period of herd closure new pigs cannot be introduced to the farm. This applies also to internal replacements of gilts to the breeding herd (Torremorell and Christianson, 2002). The success of PRRS elimination depends on the biosecurity practices and the co-operative work (Gillespie and Caroll, 2003). Consequently, one of the most important measures is to follow a strict biosecu- 
rity protocol which includes preventive actions to prevent direct and indirect routes of spread and miscellaneous routes.

The objective of this study was to eliminate PRRS from a small farrow-tofinish pig farm with herd closure, improved biosecurity and serum inoculation.

\section{Materials and methods}

Farm

The study was carried out from January 2012 to February 2015 on a farrow-to-finish farm. During the study the farm had one boar and from 42 to 62 breeding sows. Semen originated from a semen collection centre free of PRRS. At the beginning of the study the PRRS status was unknown. Reproductive failure with late abortion, irregular returns to oestrus or nonpregnant sows, stillbirth, weak piglets and formation of uneven groups at weaners were noticed. After the first serum sampling, PRRS infection was confirmed by detecting antibodypositive pigs in different categories. Serum inoculation was performed once on the farm after the confirmation of PRRS. Later follow-up samplings and laboratory testing were evaluated to confirm the elimination of PRRSV from the herd.

\section{Herd closure}

Introducing new pigs to the farm was prohibited for 200 days after serum inoculation. During this period also gilts from the farm were not allowed to be introduced into the breeding herd.

\section{Biosecurity measures}

The owner was acquainted with the obligatory measures of strict biosecurity protocols which were revised and/or introduced at the beginning of the study on the farm (personnel entering the farm only after changing clothes, coveralls and boots, extensive hand washing and footbaths, individual responsibility for each pig category, all-in/all-out system, single-age category of pigs allowed in a room, oneway pig flow, cleansing and disinfection of pens, using only equipment originating from the farm, rodent control and disinfestation). After stabilisation the breeding herd, the owner can introduce new PRRS-negative breeding pigs only via quarantine.

\section{Preparation of inoculum for serum inoculation of breeding pigs}

Twenty weaners between 8 and 14 weeks of age were bled and individually tested for PRRSV by RT-PCR method for the serum inoculum. The inoculum was prepared from PRRSV-positive serum samples by pooling of individual samples. Four parts of RPMI-1640 medium (Gibco, Germany) were added to one 
part of the pool and mixed with $1 \%$ of antibiotic-antimycotic solution (Invitrogen, Germany). All breeding pigs (62 pigs) were inoculated intramuscularly with $2 \mathrm{ml}$ of serum inoculum/pig on the same day.

\section{Sampling procedure}

At each sampling all breeding pigs and approximately five samples of each age category $(8,10,12,14$ weeks of age) and fatteners before slaughter were tested (Table 1).

A total of 630 blood samples were collected for serology and 442 for nucleic acid detection of PRRSV. Sequencing was performed from six PRRSV positive samples (Table 1).

\section{Table 1}

Number of pigs tested by serology (ELISA for the detection of PRRS antibodies), for PRRSV genome detection (RT-PCR method) and for sequencing

\begin{tabular}{|c|c|c|c|c|c|c|}
\hline \multirow[t]{2}{*}{ Sampling } & \multicolumn{2}{|c|}{$\begin{array}{c}\text { No. } \\
\text { of sera tested } \\
\text { by ELISA }\end{array}$} & \multicolumn{2}{|c|}{$\begin{array}{c}\text { No. } \\
\text { of sera tested } \\
\text { by RT-PCR }\end{array}$} & \multicolumn{2}{|c|}{$\begin{array}{c}\text { Sequencing } \\
\text { of PRRSV-positive } \\
\text { samples }\end{array}$} \\
\hline & $\begin{array}{l}\text { Breeding } \\
\text { pigs }\end{array}$ & Pigs* & $\begin{array}{l}\text { Breeding } \\
\text { pigs }\end{array}$ & Pigs* & $\begin{array}{l}\text { Breeding } \\
\text { pigs }\end{array}$ & Pigs $^{*}$ \\
\hline Confirmation of PRRS & 5 & 5 & NT & NT & NT & NT \\
\hline Before serum inoculation & 50 & 6 & 50 & 6 & NT & 2 \\
\hline 3 months after serum inoculation & 62 & NT & 62 & NT & NT & NT \\
\hline 6 months after serum inoculation & 52 & 30 & 52 & 30 & NT & 2 \\
\hline 9 months after serum inoculation & 43 & 26 & 43 & 26 & NT & 2 \\
\hline 12 months after serum inoculation & 43 & 31 & 43 & 31 & NT & NT \\
\hline 16 months after serum inoculation & 51 & 30 & 43 & 30 & NT & NT \\
\hline 20 months after serum inoculation & 42 & 26 & NT & 26 & NT & NT \\
\hline 27 months after serum inoculation & 44 & 20 & NT & NT & NT & NT \\
\hline 34 months after serum inoculation & 44 & 20 & NT & NT & NT & NT \\
\hline Total & 436 & 194 & 293 & 149 & 0 & 6 \\
\hline
\end{tabular}

${ }^{*}$ Pigs = all categories from weaning pigs to fatteners were included; NT = not tested

\section{Enzyme-linked immunosorbent assay (ELISA)}

The HerdChek, PRRS X3 ELISA test (IDEXX Laboratories) was used for detecting antibodies in serum samples. The ELISA was performed according to the manufacturer's instructions. The results of tested samples were divided into four groups; samples with an S/P lower than 0.4 (negative), samples with an S/P between 0.4 and 1 (low positive), samples with an S/P between 1 and 2 (positive), and samples with an $\mathrm{S} / \mathrm{P}$ higher than 2 (high positive). 


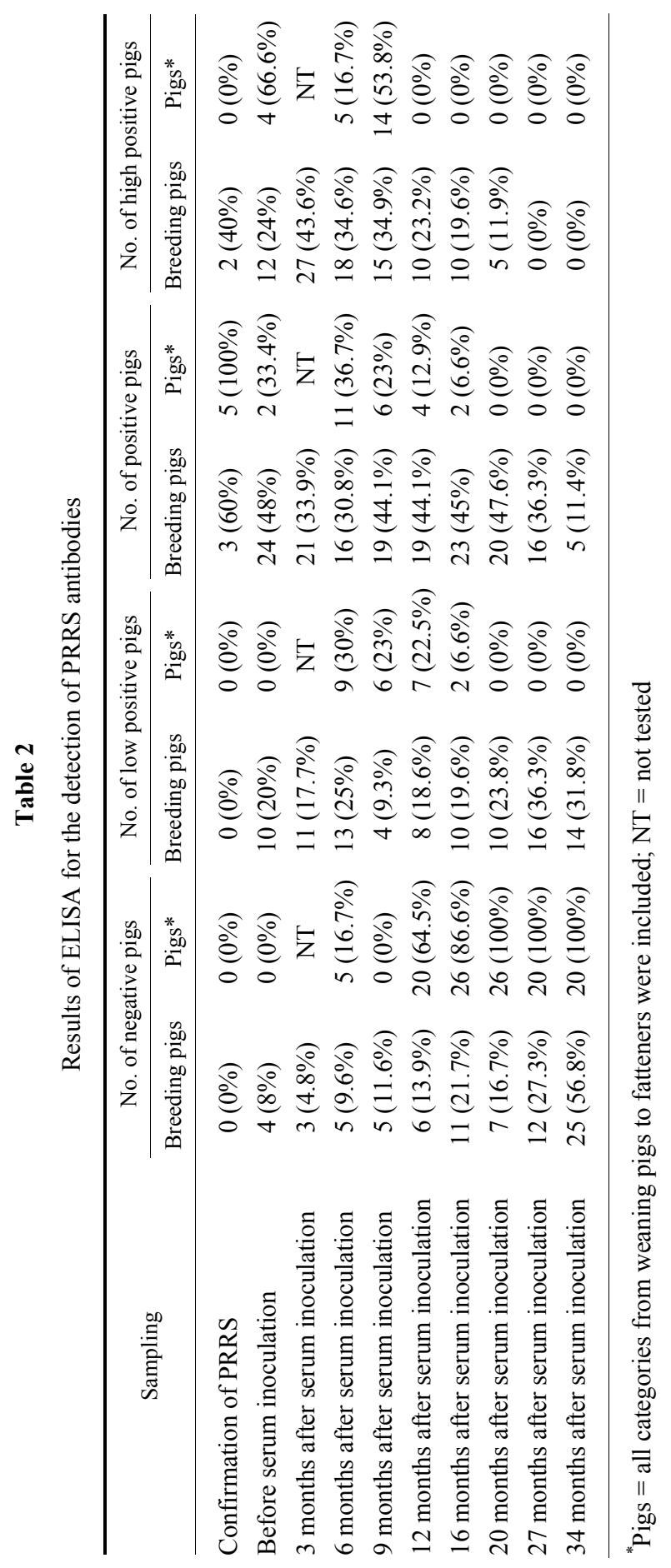


Detection of PRRSV with gel-based RT-PCR and direct Sanger sequencing of PRRSV-positive samples

Total RNA was extracted from $140 \mu$ of serum samples using the QIAamp ${ }^{\circledR}$ viral RNA mini kit (Qiagen, Germany) according to the manufacturer's instructions. A total of 442 samples were tested individually or as pools (maximum five samples in one pool) by one-step RT-PCR (One-Step RT-PCR Kit, Qiagen, Germany) using oligonucleotide primers based on the open reading frame 7 (ORF7), which detects Type 1 and Type 2 PRRSV strains (Donadeu et al., 1999; Toplak et al., 2012). The PRRSV-negative serum samples and the Lelystad virus (Type 1) were used as negative and positive controls, respectively. Six PRRSVpositive samples were directly sequenced in both directions using Sanger sequencing in the Macrogen sequencing service (Macrogen, South Korea) and the RT-PCR amplification primers. For each sample 258-nucleotide-long sequences of ORF7 were aligned with the published data using BLAST (ref. web http://www.ncbi.nlm.nih.gov/) at the National Centre for Biotechnology Information (NCBI), and PRRSV sequences obtained were compared using the sequence analysis software Lasergene ${ }^{\circledR}$ (DNASTAR Inc., Madison, WI, USA).

\section{Results}

PRRS infection on the farm was confirmed by testing five breeding pigs and five fatteners. The PRRSV infection of the herd was confirmed by the detection of a total of eight positive and two high positive pigs by ELISA (Table 2). Before serum inoculation, four samples $(8 \%)$ of breeding pigs were negative, 10 $(20 \%)$ were low positive, $24(48 \%)$ were positive and $12(24 \%)$ were high positive. After serum inoculation no more abortions, premature farrowing or increase in stillborns and mummifications were observed in the herd.

Three months after the serum inoculation three (4.8\%) previously negative breeding pigs remained negative, while $11(17.7 \%)$ breeding pigs were low positive, $21(33.9 \%)$ positive and $27(43.6 \%)$ high positive. Six months after serum inoculation the number of high positive breeding pigs decreased to $18(34.6 \%)$, the number of positive breeding pigs decreased to $16(30.8 \%)$, the number of low positive breeding pigs increased to $13(25 \%)$ and the number of negative breeding pigs increased to five $(9.6 \%)$ compared to a prior sampling. We also checked the status of 30 weaners six months after the serum inoculation: five were negative, nine low positive, 11 positive, and five high positive. Samples collected nine months after serum inoculation showed a trend of decrease in the number of low positive breeding pigs, an increasing group of positive pigs to 19 (44.2\%) and a relatively small change in the number of high positives which remained almost at the same level, 15 (34.9\%). The number of negative breeding pigs were the same as at the previous sampling. All tested weaners were still either low 
positive $(23.1 \%)$ or positive (23.1\%) and high positive (53.8\%). Twelve months after serum inoculation the number of high positive breeding pigs decreased to $10(23.3 \%)$, the number of positive remained the same, $19(44.2 \%)$ and the number of low positive increased to eight (18.6\%), as did the number of negative pigs to $6(13.9 \%)$. The majority of tested weaners were negative $(64.5 \%)$, five weaners and two fatteners $(22.6 \%)$ were low positive, and one weaner and three fatteners $(12.9 \%)$ were positive. From 20 months after serum inoculation all tested weaners and fatteners were without antibodies against PRRSV till the end of the study. The number of negative breeding pigs increased constantly and at the last sampling, 34 months after serum inoculation, the herd already consisted of 25 $(56.8 \%)$ negative breeding pigs. No more high positive breeding pigs were detected from 27 months after serum inoculation until the end of the study.

The number of high positive breeding pigs increased three months after the serum inoculation; a trend of breeding herd stabilisation was evident six months after serum inoculation. The number of high positive breeding pigs decreased nine months after serum inoculation, and this trend continued until 27 months after serum inoculation when there were no more high positive breeding pigs. Furthermore, the opposite - a trend of increase in the number of negative breeding pigs was evident from six months after serum inoculation till the end of testing (Fig. 1).

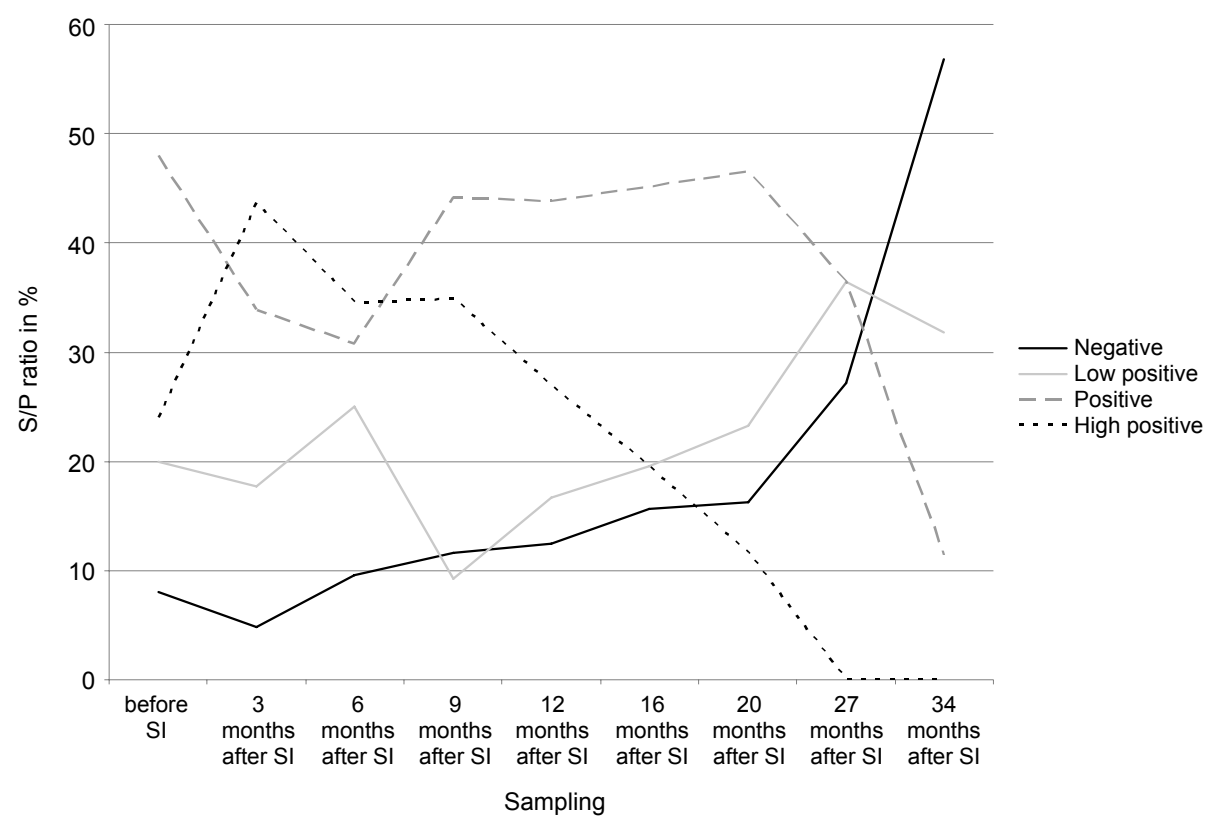

Fig. 1. Summary of serological (ELISA) results obtained in the breeding herd with presentation of changes in percent of negative, low positive, positive and high positive pigs during each sampling. Samples with an S/P lower than 0.4 were presented as negative, samples with an S/P between 0.4 and 1 as low positive, samples with an S/P between 1 and 2 as positive, and samples with an $\mathrm{S} / \mathrm{P}$ higher than 2 as high positive. SI: serum inoculation 
During the complete period of study, all tested pigs in the breeding herd were negative for PRRSV by RT-PCR method at all samplings, while weaners and growers aged 6 and 12 weeks were positive until nine months after serum inoculation. At the last three samplings (12, 16 and 20 months after serum inoculation) all pigs were PRRSV negative by RT-PCR (Table 3). The observed nucleotide homology between six PRRSV-positive samples obtained from weaners (positive samples collected before serum inoculation, 6 months and 9 months after serum inoculation) based on comparison of the 258-nucleotide-long partial sequence of ORF7 was $99.6-100 \%$, confirming that only one strain of PRRSV was circulating in the herd during the period of study. The detected PRRSV strain in the infected herd belonged to Type 1 and was clustered into a lineage named 1n. The detected PRRSV shared 93.0-93.4\% nucleotide identity with the Lelystad virus. The closest sequences identified in GenBank were strain Kre which was detected in Lithuania (KC713975), strain CRO detected in Croatia (KF498724) and strain DK-2012-01-05-11 detected in Denmark (KC862548). These three strains share only $94 \%$ of nucleotide identity with the PRRSV strain collected from the infected pig herd in this study.

Table 3

Results of the RT-PCR

\begin{tabular}{llllllc}
\hline \multirow{2}{*}{ Sampling } & \multicolumn{7}{c}{ Results } \\
\cline { 2 - 7 } & $\begin{array}{c}\text { Breeding } \\
\text { pigs }\end{array}$ & $\begin{array}{c}\text { Pigs 6 } \\
\text { weeks }\end{array}$ & $\begin{array}{c}\text { Pigs 8 } \\
\text { weeks }\end{array}$ & $\begin{array}{c}\text { Pigs 10 } \\
\text { weeks }\end{array}$ & $\begin{array}{c}\text { Pigs 12 } \\
\text { weeks }\end{array}$ & $\begin{array}{c}\text { Pigs 14 } \\
\text { weeks }\end{array}$ \\
\hline Before serum inoculation & NT & pos. & pos. & pos. & pos. & NT \\
3 months after serum inoculation & neg. & NT & NT & NT & NT & NT \\
6 months after serum inoculation & neg. & neg. & neg. & pos. & neg. & neg. \\
9 months after serum inoculation & neg. & neg. & neg. & pos. & pos. & neg. \\
12 months after serum inoculation & neg. & neg. & neg. & neg. & neg. & neg. \\
16 months after serum inoculation & neg. & neg. & neg. & neg. & neg. & neg. \\
20 months after serum inoculation & neg. & NT & NT & neg. & neg. & neg. \\
27 months after serum inoculation & NT & NT & NT & NT & NT & NT \\
34 months after serum inoculation & NT & NT & NT & NT & NT & NT \\
\hline
\end{tabular}

neg. $=$ PRRSV negative; pos. $=$ PRRSV positive; $\mathrm{NT}=$ not tested

\section{Discussion}

Considering the heterogeneity of PRRS serotypes and the importance of homologous immunity, serum inoculation can be a successful measure for elimination of PRRS from the farm (McCawe, 2006). PRRSV successfully circulates in endemically infected herds until at any given time enough pigs are in various 
stages of infection and immunity (Dee et al., 1996). For the successful elimination of PRRSV in an infected herd, the first replication of the virus within a population of breeding pigs must stop in order to prevent vertical spread of the infection (Zimmerman et al., 2012).

From the beginning of the study we implemented herd closure to prevent introduction of a new virus strain into the infected herd. PRRSV elimination through herd closure is based on the fact that naturally developed immunity eliminates virus infection from the farm (Torremorell and Christianson, 2002). Using serum inoculation we tried to enhance the development of homologous immunity which was confirmed with improved protection according to the results of serology. The results of serology three months after the serum inoculation showed a dramatic increase in the number of high positive breeding pigs, suggesting a good immune response in pigs after serum inoculation. Six months after the serum inoculation, a trend of breeding herd stabilisation was visible, supporting also the observations of other authors (Otake et al., 2002; Dee, 2004; Zimmerman et al., 2012). From 9 months until 27 months after serum inoculation a steady decrease of high positive breeding pigs was noticed and at the last two samplings, there were no more high positive pigs in the breeding herd. The increasing percentage of negative breeding pigs detected at the last samplings confirmed the elimination of PRRSV from the herd. The same five negative breeding pigs remained negative 6,9 , and 12 months after serum inoculation and stayed negative until the end of the study, which additionally indicated that the circulation of PRRSV in breeding pigs had ceased soon after serum inoculation. No more seropositive weaners and fatteners were detected 20 months after serum inoculation, confirming also the elimination of PRRSV from younger pigs in the herd. The persistence of antibodies for at least 34 months after serum inoculation is not consistent with the data of Zimmerman et al. (2012), who reported a decline of antibodies to a negative level 12 months after infection. From the obtained data of intermittent detection of PRRSV in groups of weaners until 12 months after serum inoculation, we can speculate that PRRSV was occasionally transmitted to the breeding herd, which resulted in a prolonged period of antibody detection.

Twelve months after serum inoculation all samples, collected from weaners, growers and fatteners, were PRRSV negative. This confirmed that the elimination of PRRSV from weaners, growers and fatteners needs time and probably varies in different infected herds. Partial depopulation and the test and removal strategy can decrease the time needed for the elimination of PRRSV from a herd. The previous observation for Slovenia was that PRRSV was endemically present in swine across the whole territory as a result of low biosecurity standards, intensive trading and importation of pigs from different countries (Toplak et al., 2012). According to the results of the RT-PCR method and direct sequencing of six PRRSV-positive samples, we confirmed that during the entire period of study the same strain of PRRSV was present, and that herd closure and other preven- 
tive biosecurity measurements successfully prevent the introduction of new PRRSV strains into the herd. Although only partial sequencing $(258 \mathrm{nt})$ was done for six PRRSV positive samples in ORF7 in this particular study, we can confirm that only one PRRSV strain was circulating in the infected herd during the study. Sequences of six positive samples were compared to the national PRRSV sequence data collection consisting of about 300 sequences from 150 positive herds, and a closely related strain ( $98.4 \%$ nucleotide identity in ORF7) was detected in only one other PRRSV-infected herd (data not shown). Sequencing of ORF5 was not done for this almost unique PRRSV strain detected only in two herds in Slovenia; nonetheless, such sequencing could be important for the phylogenetic comparison of PRRSVs circulating in Europe.

At every visit we checked the implementation of biosecurity measures according to our written guidelines. On the basis of both the owner's assurance and our check-up, all required measures were followed. The owner introduced his own replacement gilts before the serum inoculation (load), which was also seen by other authors (Rowland and Morrison, 2012). Another very important measure is the allin/all-out protocol, which was followed as required. Personnel were appointed to a single pig category and changed coveralls and boots between pig categories. In addition, they washed their hands between pig categories. The implementation of the required biosecurity measures as well as the execution of strict herd closure are necessary for the successful elimination of PRRSV from farms (Otake et al., 2002; Zimmerman et al., 2012). Elimination of PRRS from farms can be achieved earlier if additional measures such as immunisation of breeding pigs and partial depopulation are implemented. Dee et al. (1993) reported that partial depopulation and strict biosecurity measures can stop the circulation of PRRSV in weaners.

Serum inoculation of breeding pigs and the implementation of herd closure and biosecurity measures proved to be a successful measure for the elimination of PRRS from the farrow-to-finish farm despite the fact that a good candidate for the elimination of PRRS is the three-site farm. Moreover, the success rate was reported to be above $85 \%$ for farms with segregated production (Torremorell and Christianson, 2002).

\section{References}

Batista, L., Pijoan, C. and Torremorell, M. J. (2002): Experimental infection of gilts with porcine reproductive and respiratory syndrome virus (PRRSV) during acclimatization. J. Swine Health Prod. 10, 147-150.

Dee, S. A. (2004): Elimination of porcine reproductive and respiratory syndrome virus from 30 farms by test and removal. J. Swine Health Prod. 12, 129-133.

Dee, S. A., Bierk, M. D., Deen, J. and Molitor, T. W. (2001): An evaluation of test and removal for the elimination of PRRS virus from infected breeding herds. Can. J. Vet. Res. 65, 22-27.

Dee, S. A., Joo, H. S., Tokach, L., Park, B. K., Molitor, T. W. and Pijoan, C. J. (1996): Detecting subpopulations after PRRS virus infection in large breeding herds using multiple serologic tests. J. Swine Health Prod. 4, 181-184. 
Dee, S. A., Morrison, R. B. and Joo, H. S. J. (1993): Eradicating porcine reproductive and respiratory syndrome (PRRS) virus using two-site production and nursery depopulation. J. Swine Health Prod. 5, 20-23.

Donadeu, M., Arias, M., Gomez-Tejedor, C., Aguero, M., Romero, L. J., Christianson, W. T. and Sanchez-Vizcaino, J. M. J. (1999): Using polymerase chain reaction to obtain virus PRRSfree piglets from epidemically infected herds. J. Swine Health Prod. 7, 255-261.

Duinhof, T. F., Houben, M., Nes, A. and Hanssen, M. (2014): PRRS regional control in Europe: the Dutch project is one year on the road. Pig 333.com https://www.pig333.com/print/8353 (30.5.2015)

Fano, E., Olea, L. and Pijoan, C. (2005): Eradication of porcine reproductive and respiratory syndrome virus by serum inoculation of naïve gilts. Can. J. Vet. Res. 69, 71-74.

Gillespie, T. G. and Caroll, A. L. (2003): Techniques for PRRSV elimination utilizing modified live virus vaccines on single-site swine farms. Proceedings of the 4th International Symposium on Emerging and Re-emerging Pig Diseases, 29 June - 2 July, Rome, Italy, pp. 127-128.

Gramer, M. L., Christianson, W. T. and Harris, D. L. (1999): Producing PRRS negative pigs from PRRS positive sows. Proceedings of the American Association of Swine Practitioners, Annual Meeting. Lousiana, ZDA, pp. 413-416.

Holtkamp, D. J., Kliebenstein, J. B., Neumann, E. J., Zimmerman, J. J., Rotto, H. F., Yoder, T. K., Wang, C., Yeske, P. E., Mowrer, C. L. and Haley, C. (2013): Assessment of the economic impact of porcine reproductive and respiratory syndrome virus on United State pork producers. J. Swine Health Prod. 21, 72-84.

Kimman, T. G., Cornelissen, L. A., Moormann, R. J., Rebel, J. M. J. and Stockhofe-Zurwieden, N. S. (2009): Challenges for porcine reproductive and respiratory syndrome virus (PRRSV) vaccinology. Vaccine 27, 3704-3718.

McCawe, M. (2006): Different approaches to handling PRRS. Proceedings of London Swine Conference - Thinking Globally, Acting Locally. 5-6 April, London, Great Britain, pp. 21-33.

Meng, X. J. (2000): Heterogeneity of porcine reproductive and respiratory syndrome virus: implications for current vaccine efficacy and future vaccine development. Vet. Microbiol. 74, 309-329.

Murtaugh, M. P. and Genzow, M. (2011): Immunological solutions for treatment and prevention of porcine reproductive and respiratory syndrome (PRRS). Vaccine 29, 8192-8204.

Otake, S., Dee, S. A., Rossow, K. D., Deen, J., Joo, H. S., Molitor, T. W. and Pijoan, C. (2002): Transmission of porcine reproductive and respiratory syndrome virus by fomites (boots and coveralls). J. Swine Health Prod. 10, 59-65.

Rajic, A., Dewey, C. E., Deckert, A. E., Friendship, R. M., Martin, S. W. and Yaoo, D. J. (2001): Production of PRRSV-negative pigs commingled from multiple, vaccinated, serologically stable, PRRSV-positive breeding herds. Swine Health Prod. 9, 179-184.

Rowland, R. and Morrison, R. (2012): Challenges and opportunities for the control and elimination of porcine reproductive and respiratory syndrome virus. Transbound. Emerg. Dis. 59, 55-59.

Shibata, I., Mori, M. and Yazawa, S. J. (2000): Experimental reinfection with homologous porcine reproductive and respiratory syndrome virus in SPF pigs. Vet. Med. Sci. 62, 105-108.

Toma, B., Vaillancourt, J. P., Dufour, B., Eloit, M., Moutou, F., Marsh, W., Benet, J. J., Sanaa, M. and Michel, P. (1991): Dictionary of Veterinary Epidemiology. State University Press, Ames, Iowa. p. 83.

Toplak, I., Rihtarič, D., Hostnik, P., Grom, J., Štukelj, M. and Valenčak, Z. (2012): Identification of a genetically diverse sequence of porcine reproductive and respiratory syndrome virus in Slovenia and the impact on the sensitivity of four molecular tests. J. Virol. Meth. 179, 51-56.

Torremorell, M. and Christianson, W. T. (2002): PRRS eradication by herd closure. Advanc. Pork Prod. 13, 169-176.

Zimmerman, J., Benefield, D. A., Murtaugh, M. P., Osorio, F., Stevenson, G. W. and Torremorell, M. (2012): Porcine reproductive and respiratory syndrome virus (Porcine Arterivirus). In: Zimmerman, J. J., Karriker, L. A., Ramirez, K. J. and Stevenson, G. W. (eds) Diseases of Swine. 10th edition. Blackwell Publishing Professional, Ames, Iowa. pp. 461-486. 